\title{
Article \\ Factors Related to Psychological Distress during the First Stage of the COVID-19 Pandemic on the Chilean Population
}

\author{
Carlos Ruiz-Frutos ${ }^{1,2}\left(\mathbb{D}\right.$, Diemen Delgado-García ${ }^{3}$, Mónica Ortega-Moreno ${ }^{4, *}$, , Daniel Duclos-Bastías ${ }^{5}{ }^{(1)}$, \\ Dánica Escobar-Gómez ${ }^{5,6}$, Juan Jesús García-Iglesias ${ }^{1}$ (1) and Juan Gómez-Salgado ${ }^{1,2, * \mathbb{C}}$
}

1 Department of Sociology, Social Work and Public Health, Faculty of Labour Sciences, University of Huelva, 21007 Huelva, Spain; frutos@uhu.es (C.R.-F.); juanjesus.garcia@dstso.uhu.es (J.J.G.-I.)

2 Safety and Health Postgraduate Programme, Universidad Espíritu Santo, Guayaquil 092301, Ecuador

3 IMPACTCOVID-19 Chile Research Project, Universidad de Aconcagua, Los Andes 2102660, Chile; diemen.delgado@uac.cl

4 Department of Economy, University of Huelva, 21007 Huelva, Spain

5 School of Physical Education, Faculty of Philosophy and Education, Pontificia Universidad Católica de Valparaíso, Valparaíso 2374631, Chile; daniel.duclos@pucv.cl (D.D.-B.); danica.escobar@pucv.cl (D.E.-G.)

6 Faculty of Education Sciences, University of Cádiz, 11519 Cádiz, Spain

* Correspondence: ortegamo@uhu.es (M.O.-M.); salgado@uhu.es (J.G.-S.); Tel.: +34-959219700 (J.G.-S.)

Citation: Ruiz-Frutos, C.;

Delgado-García, D.; Ortega-Moreno,

M.; Duclos-Bastías, D.;

Escobar-Gómez, D.; García-Iglesias,

J.J.; Gómez-Salgado, J. Factors Related

to Psychological Distress during the

First Stage of the COVID-19

Pandemic on the Chilean Population.

J. Clin. Med. 2021, 10, 5137. https://

doi.org/10.3390/jcm10215137

Academic Editors: Giacomo Mancini and Michele Roccella

Received: 22 September 2021

Accepted: 29 October 2021

Published: 31 October 2021

Publisher's Note: MDPI stays neutral with regard to jurisdictional claims in published maps and institutional affiliations.

Copyright: (c) 2021 by the authors. Licensee MDPI, Basel, Switzerland. This article is an open access article distributed under the terms and conditions of the Creative Commons Attribution (CC BY) license (https:/ / creativecommons.org/licenses/by/ $4.0 /)$.
Abstract: The health effects of COVID-19 continue to raise doubts today. In some areas, such as mental health, these doubts have scarcely been addressed. The present study analyses the effects on psychological distress during the first phase of the pandemic in Chile. A cross-sectional descriptive study was performed by using a questionnaire validated in Spain and adapted for Chile. Between 22 April and 16 December 2020, 3227 questionnaires were collected from the 16 regions of Chile, using non-probabilistic snowball sampling. Bivariate analysis and binary logistic regression were performed. The variables that could predict psychological distress during the COVID-19 pandemic in Chile were: having a poor self-perception of health $\mathrm{OR}=4.038,95 \% \mathrm{CI}=(2.831,5.758)$; being younger than $29 \mathrm{OR}=2.287,95 \% \mathrm{CI}=(1.893,2.762)$; having diarrhea $\mathrm{OR}=2.093,95 \% \mathrm{CI}=(1.414,3.098)$; having headache $\mathrm{OR}=2.019,95 \% \mathrm{CI}=(1.662,2.453)$; being a woman $\mathrm{OR}=1.638,95 \% \mathrm{CI}=(1.363$, $1.967)$; having muscle pain $\mathrm{OR}=1.439,95 \% \mathrm{CI}=(1.114,1.859)$; and having had casual contact with an infected person $\mathrm{OR}=1.410,95 \% \mathrm{CI}=(1.138,1.747)$. In Chile, with a better social, economic, cultural, and health environment compared to neighboring countries, there has been a high percentage of psychological distress. It is time to prioritize measures to safeguard the mental health of Chileans, especially focused on the most vulnerable population according to our results, i.e., young women with poorer health status.

Keywords: COVID-19 emergency; psychological distress; stress disorder; preventive measures; mental health; Chile

\section{Introduction}

COVID-19, with an onset at the end of 2019 in Wuhan, China, was declared by the WHO as an international public health emergency in January 2020 [1] and as a global pandemic in March 2020 [2]. It spread rapidly throughout Latin American countries, leading the WHO to declare the region as an epicenter of the pandemic in May 2020 [3].

It has been estimated that in Latin American countries, including Chile, despite the fact that preventive measures against COVID-19 were implemented without delay, these have not had the expected effects due to, among other causes, deficiencies in the contact tracking and follow-up system, as well as problems prior to the pandemic, such as the characteristics of the health system, social inequalities, high rates of informal employment, and little or late establishment of economic support measures [4].

Regarding health inequities, proven to exist in Chile, and the finding of higher mortality rates in the metropolitan area of Chile, observing a direct association between mortality 
from COVID-19 and poverty [5]. The social determinants of health, in particular the multidimensional poverty index and the use of public transport, play an important role in explaining the differences in outcomes [6], both in the incidence of COVID-19 and in mortality [7].

On the other hand, the influence of the economy on health is an aspect of special relevance. In this regard, analyses of the effects of the containment measures on Chile's economic activity have been carried out [8]. One of these analyses, that covered the response to the pandemic by several Latin American governments, including Chile, has found that while wealthier municipalities introduce technological innovations comparable to those in developed countries, smaller or less advantaged areas have more difficulty maintaining service delivery while in an unprecedented socio-economic context, as is the one experienced during the pandemic [9]. However, it has been found that this health situation has not affected all population sectors in Chile equally [10]. Thus, as for the indigenous population, the vulnerability indicators previously detected have increased since it is a group that already had inequalities in health [11,12].

Overall, the impact of the COVID-19 pandemic in Chile has been significant. According to official data, as of 19 May 2021, 1,292,096 cases had been confirmed (6.81\% of the general population), with 27,934 deaths and $39.78 \%$ of the country's total population fully vaccinated. This last figure is much higher than in the countries that surround Chile as well as some of the European continent, such as Spain [13].

Regarding the effects of the physiological symptoms of the disease, cough, dyspnea, anosmia, generalized fatigue, and respiratory type problems predominate, as well as an increased risk of thromboembolic events as a result of the inflammatory state generated by the cytokine storm [14-17], although many infected persons remain asymptomatic [18]. As for the adoption of preventive measures, it has been shown that they have contributed to reducing the impact of the pandemic in those countries where they have been adopted early, with Chile having carried out a high number of diagnostic tests [19]. Several studies have been carried out to determine the factors that influence the use of preventive measures to prevent COVID-19 and their association with the development of psychological distress. It has been determined that "hand washing" was the most widely used preventive measure in Spain during the first phase of the pandemic [20], that preventive measures will depend on the risk perception acquired [21], and that psychological distress depends on the high perceived costs of adherence to the preventive measures [22], uncomfortable feeling of wearing personal protective equipment, or the public ignorance of preventive measures [23].

One of the main characteristics of the COVID-19 pandemic is that it has been classified as a "psychological pandemic", with great effects on the mental health of the general population and, especially, on health professionals, who have been directly involved in the care of patients with COVID-19 [16,24-26]. In this sense, greater psychological affectation has been shown in professionals who were quarantined, who worked in COVID-19 units, or had a family member or friend infected with COVID-19. These effects manifested more through greater depression, anxiety, frustration, fear, and post-traumatic stress than in those persons who did not have such experience [27].

Regarding the general population, women [24], young people, the self-employed, individuals with previous psychological issues whose follow-up was interrupted [28], immigrants, or workers of essential activities and in contact with the public were the most affected strata $[26,29]$. Higher levels of stress, depression, or anxiety have been found in these groups [15,24,30-32], as well as somatization [24] and psychiatric disorders [17], especially in those patients with previous mental problems [33,34]. However, a study conducted in 21 countries, including Chile, did not find an increase in the number of suicides in the first months of the pandemic [35]. The psychological impact of the pandemic has manifested itself even in countries with low infection rates and good initial management of the outbreak, such as South Korea. In this population, symptoms of stress, anxiety, 
depression, and sleeping difficulties have been reported, albeit to a minimal or moderate degree. [36].

Among these findings, it is noteworthy that, although older people have a higher risk of suffering from serious illness due to COVID-19, they show fewer negative effects on their emotional health than young people [37], something also proven in other countries [38]. However, the elderly population is more vulnerable to stigma related to COVID-19. As an at-risk population, they are known to be more likely to be affected by the disease and this can lead to stigmatization, resulting in social rejection, isolation, and discrimination [39].

These works, carried out at the international level, provide an overview of the state of mental health among the population during the COVID-19 pandemic. However, there is currently no data that records this situation in Chile, that is, the psychological impact of the pandemic on the Chilean population has not been described. Thus, the novelty of this work lies in being the first to study this problem in Chile. The results would help measure and describe the impact of the pandemic, guide strategies for managing and addressing the crisis, and design interventions adapted to the needs of the Chilean population, as well as to develop a prevention plan for similar future situations.

Therefore, the objective of this article is to present the effects of the first wave of the COVID-19 pandemic on the mental health of Chileans, in particular, in the development of psychological distress. In this sense, it is intended to analyze the possible association with sociodemographic variables, perception of health, physical symptoms, having required health care, having received diagnostic tests, adoption of preventive measures, or contact history, among others.

\section{Materials and Methods}

\subsection{Design and Sample}

The design was a cross-sectional descriptive study, using a questionnaire previously validated and cross-culturally adapted to the Chilean environment. The sample was made up of the Chilean population, accessed through the non-probability sampling methodology snowballing method, the same methodology used in the study carried out in "Europe on Living, Working and COVID-19" by Eurofound [40].

In order to participate in the study, it was necessary to meet the following inclusion criteria: reside in Chile, be over 18 years old, and accept the informed consent. The estimated sample size was 3294, with $95 \%$ confidence level, a precision of $1.8 \%$ and a loss adjustment of $10 \%$. Finally, the loss was $8.14 \%$, leaving a sample size of 3227 .

\subsection{Materials}

A questionnaire previously validated in Spain [20] was used, composed of several previously validated instruments adapted to the linguistic and cultural use of the language in Chile so that no question posed any difficulty of understanding. For this purpose, a panel of experts consisting of psychologists, epidemiologists, doctors, nurses, and public health experts was selected.

The questionnaire consists of three parts. In the first part, sociodemographic data were included: sex, level of education, age, work situation, cohabitation, having children or pets at home, having some degree of disability, and being under lockdown at home. In the second, Goldberg's general health questionnaire (GHQ-12) [41] was used to measure the level of mental health and psychological well-being. This questionnaire consisted of 12 items and four answer options, in which 1 meant better than usual or more than usual, 2 same as usual, 3 less than usual or less so, and 4 much less than usual or much less, as regards the positive items. As for the negative ones, 1 meant not at all, 2 no more than usual, 3 rather more than usual or rather more, and 4 much more than usual or much more. 0 points were assigned to the first two options, and 1 point to the last two, with a total score ranging from 0 to 12 . The cut-off point established for the general population was three, considering scores greater than or equal to 3 as psychological distress. In the third part, questions were related to the perception of COVID-19 symptoms and the history of contacts during the 
last 14 days: headache or sore throat, cough, fever, rhinitis, dizziness, myalgia, shortness of breath, chills, or diarrhea. Questions about taking medication, suffering from chronic illness, or having required medical attention or hospitalization during the last 14 days were also included. These items were assessed with a yes/no dichotomous answer. The possible history of contact during the last 14 days was measured by means of three items: possible contact for more than 15 min less than two meters away, casual contact with confirmed infected persons, and contact with persons or materials suspected of being infected; also, the existence of an infected relative diagnosed by a diagnostic test. Participants could respond categorically to these items with three possible options: yes, no, or doesn't know.

Another variable collected was self-perception of their level of health during the last two weeks, this being a well-known indicator for predicting mortality [42]. It was measured with five levels of response, from very bad to very good, grouped for the final analysis into two categories, bad and excellent.

Finally, the preventive measures adopted were also included in the questions, using a Likert scale with five response options categorized from never to always with respect to the frequency with which they were performed: wearing a mask regardless of the presence or absence of symptoms; washing hands immediately after coughing, touching the nose, or sneezing; washing hands after touching potentially contaminated objects; washing hands with hydroalcoholic solution; washing hands with soap and water; covering the mouth with the elbow when coughing or sneezing; avoiding sharing utensils (e.g., spoon) during meals; leaving at least a meter and a half of separation from others. One point was assigned to the never answer, 2 points to rarely, 3 points to sometimes, 4 to almost always, and 5 to always. Thus, each item could score between 1 and 5 , and the total score of the scale would range from 8 to 40 .

\subsection{Procedure}

The Qualtrics ${ }^{\circledR}$ storage and survey platform (Qualtrics, Provo, UT, USA) was used to collect the information through an online questionnaire. For its dissemination, the collaboration of universities and scientific societies was requested, and social networks and interviews in the press were used. The questionnaire was disseminated online and through the social media in order to reach a larger number of participants.

\subsection{Data Analysis}

Frequencies, means and/or standard deviations were presented depending on the type of variable. The relationship of the qualitative variables with the psychological distress was analyzed through the Chi-squared test, also obtaining the odds ratio (OR) with the associated confidence intervals. The association between the different scores was analyzed by the Student's $t$-test for independent samples.

Finally, a binary logistic regression was performed that allowed for an assessment model to be built to study the presence or absence of psychological distress and identify those variables that played a relevant role. OR values indicate the strength of the relationship with psychological distress; the further away from 1, the stronger the relationship is.

To verify the appropriateness of the model, different measures of goodness of fit were used: the Hosmer-Lemeshow test, percentage of correctly classified values, sensitivity, and specificity. The inclusion of the variables was carried out with tests of statistical significance, the OR were estimated, and the confidence intervals were facilitated. The OR values indicate the strength of the relationship with psychological distress. All analyses were carried out with the SPSS 26.0 statistical software (IBM, New York, NY, USA).

\subsection{Ethical Principles}

At the beginning of the questionnaire, participants received information about the objectives of the study and were asked to provide their written informed consent prior to answering. The data were recorded anonymously, treated confidentially and met the 
ethical principles established in the Declaration of Helsinki (Fortaleza, 2013) and all the legal regulations in force on data protection and regulation of human research processes in Chile. The study has been authorized by the Ethics Committee of the University of Aconcagua in Chile (Santiago, UAC-22 April 2020) and in Spain by the Research Ethics Committee of Huelva, belonging to the Regional Ministry of Health of Andalusia (PI 036/20).

\section{Results}

\subsection{Sociodemographic Data}

A pilot test was carried out with 57 people, diverse regarding their profession, educational level, geographical scope, age, and sex, and where a Cronbach's alpha coefficient of 0.910 was obtained, good psychometric properties, and no understanding problems. Questionnaires were received from the 16 Regions of Chile, with higher response rates from Valparaiso and Santiago. Records were obtained from 38 of the 43 types of classified occupations, with $21 \%$ of health professionals. A total of 3227 questionnaires were received between 21 April and 24 December 2020.

As can be seen in Table 1, there is a higher percentage of women $(63.40 \%)$, people with university level education or higher studies (59.25\%), young people (51\% being 29 years old or less), living without a partner $(70.31 \%)$, without children $(63.43 \%)$, living in a house with an exterior view (house with balcony, terrace, yard, or garden) $(86.6 \%)$, having a pet $(71.4 \%)$, working in private companies $(46.27 \%)$, being health professionals $(21.2 \%)$, being in strict confinement or in confinement except for purchase-work $(81.7 \%)$.

Table 1. Association between sociodemographic variables and psychological distress during the COVID-19 pandemic.

\begin{tabular}{|c|c|c|c|c|c|c|}
\hline \multicolumn{7}{|c|}{ TOTAL $(N=3227)$} \\
\hline & \multicolumn{4}{|c|}{ GHQ } & \multirow[b]{2}{*}{$p$} & \multirow[b]{2}{*}{$\begin{array}{l}\text { Odds Ratio } \\
\text { (CI 95\%) }\end{array}$} \\
\hline & $N(\%)$ & $\begin{array}{c}\text { YES } \\
(N=2544)\end{array}$ & $\begin{array}{c}\text { NO } \\
(N=683)\end{array}$ & $x^{2}$ & & \\
\hline $\begin{array}{l}\text { Sex } \\
\text { Female } \\
\text { Male }\end{array}$ & $\begin{array}{l}2045(63.4) \\
1182(36.6)\end{array}$ & $\begin{array}{l}82.9 \\
71.7\end{array}$ & $\begin{array}{l}17.1 \\
28.3\end{array}$ & 56.224 & $<0.001$ & $\begin{array}{c}1.916 \\
(1.613,2.273)\end{array}$ \\
\hline $\begin{array}{l}\text { Age }^{*}(N=3224) \\
29 \text { years or younger } \\
\text { Older than } 29\end{array}$ & $\begin{array}{l}1654(51.30) \\
1570(48.70)\end{array}$ & $\begin{array}{l}86.5 \\
70.8\end{array}$ & $\begin{array}{l}13.5 \\
29.2\end{array}$ & 119.842 & $<0.001$ & $\begin{array}{c}2.651 \\
(2.219,3.168)\end{array}$ \\
\hline $\begin{array}{l}\text { Marital status } \\
\text { Single } \\
\text { With a couple }\end{array}$ & $\begin{array}{c}2269(70.31) \\
958(29.69)\end{array}$ & $\begin{array}{l}82.4 \\
70.5\end{array}$ & $\begin{array}{l}17.6 \\
29.5\end{array}$ & 57.282 & $<0.001$ & $\begin{array}{c}1.959 \\
(1.643,2.336)\end{array}$ \\
\hline $\begin{array}{l}\text { Educational level } \\
\text { Upper secondary school or lower } \\
\text { University or higher }\end{array}$ & $\begin{array}{l}1315(40.75) \\
1912(59.25)\end{array}$ & $\begin{array}{l}85.9 \\
74.0\end{array}$ & $\begin{array}{l}14.1 \\
26.0\end{array}$ & 65.563 & $<0.001$ & $\begin{array}{c}2.132 \\
(1.771,2.567)\end{array}$ \\
\hline $\begin{array}{c}\text { Type of dwelling } \\
\text { Apartment/House without } \\
\text { balcony/terrace/yard } \\
\text { Apartment/House with } \\
\text { balcony/terrace/yard/garden }\end{array}$ & $2796(86.6)$ & 77.9 & 22.1 & 11.893 & 0.001 & $\begin{array}{c}1.631 \\
(1.232,2.160)\end{array}$ \\
\hline $\begin{array}{c}\text { You are }(N=1502) \\
\text { Independent worker } \\
\text { Public employer } \\
\text { Worker for private comp. }\end{array}$ & $\begin{array}{l}233(15.51) \\
574(38.22) \\
695(46.27)\end{array}$ & $\begin{array}{l}61.8 \\
78.7 \\
70.6\end{array}$ & $\begin{array}{l}38.2 \\
21.3 \\
29.4\end{array}$ & 25.712 & $<0.001$ & - \\
\hline
\end{tabular}


Table 1. Cont.

\begin{tabular}{|c|c|c|c|c|c|c|}
\hline \multicolumn{7}{|c|}{ TOTAL $(N=3227)$} \\
\hline & \multicolumn{4}{|c|}{ GHQ } & \multirow[b]{2}{*}{$p$} & \multirow[b]{2}{*}{$\begin{array}{c}\text { Odds Ratio } \\
\text { (CI 95\%) }\end{array}$} \\
\hline & $N(\%)$ & $\begin{array}{c}\text { YES } \\
(N=2544)\end{array}$ & $\begin{array}{c}\text { NO } \\
(N=683)\end{array}$ & $x^{2}$ & & \\
\hline $\begin{array}{c}\text { Children } \\
\text { No } \\
\text { Yes }\end{array}$ & $\begin{array}{l}2047(63.43) \\
1180(36.57)\end{array}$ & $\begin{array}{l}83.9 \\
70.1\end{array}$ & $\begin{array}{l}16.1 \\
29.9\end{array}$ & 85.359 & $<0.001$ & $\begin{array}{c}2.222 \\
(1.873,2.639)\end{array}$ \\
\hline $\begin{array}{l}\text { Pet } \\
\text { Yes } \\
\text { No }\end{array}$ & $\begin{array}{c}2304(71.40) \\
923(28.60)\end{array}$ & $\begin{array}{l}79.2 \\
77.9\end{array}$ & $\begin{array}{l}20.8 \\
22.1\end{array}$ & 0.680 & 0.410 & $\begin{array}{c}1.081 \\
(0.898,1.301)\end{array}$ \\
\hline $\begin{array}{c}\text { Disability } \\
\text { No } \\
\text { Yes }\end{array}$ & $\begin{array}{c}3132(97.06) \\
95(2.94)\end{array}$ & $\begin{array}{l}79.0 \\
73.7\end{array}$ & $\begin{array}{l}21.0 \\
26.3\end{array}$ & 1.556 & 0.212 & $\begin{array}{c}1.342 \\
(0.844,2.137)\end{array}$ \\
\hline $\begin{array}{c}\text { Health worker } \\
\text { No } \\
\text { Yes }\end{array}$ & $\begin{array}{c}2543(78.8) \\
684(21.2)\end{array}$ & $\begin{array}{l}79.8 \\
75.3\end{array}$ & $\begin{array}{l}20.2 \\
24.7\end{array}$ & 6.528 & 0.011 & $\begin{array}{c}1.385 \\
(1.062,1.580)\end{array}$ \\
\hline $\begin{array}{c}\text { Confinement } \\
\text { Strict } \\
\text { Except for purchase-work } \\
\text { No } \\
\text { Other situations }\end{array}$ & $\begin{array}{c}843(26.1) \\
1793(55.6) \\
326(10.1) \\
265(8.2)\end{array}$ & $\begin{array}{l}81.0 \\
80.0 \\
69.0 \\
75.8\end{array}$ & $\begin{array}{l}19.0 \\
20.0 \\
31.0 \\
24.2\end{array}$ & 24.200 & $<0.001$ & - \\
\hline
\end{tabular}

\subsection{Psychological Distress}

$78.83 \%$ of the sample had psychological distress (PD), following the $\geq 3$ cut-off point of the GHQ-12. The overall mean of the 12 items (GHQ-12) was $\mathrm{M}=6.16(\mathrm{SD}=3.76)$, with a reliability coefficient of the optimal measurement scale of Cronbach's $\alpha=0.910$ (Table 2).

Table 2. Psychological Distress: General Health Questionnaire GHQ-12.

\begin{tabular}{cc}
\hline & TOTAL \\
Item & (N = 3227) \\
\hline 1. Have you been able to concentrate well on what you were doing? & M (SD) \\
2. Have your worries made you lose a lot of sleep? & $2.86(0.80)$ \\
3. Have you felt that you are playing a useful role in life? & $2.82(0.99)$ \\
4. Have you felt capable of making decisions? & $2.36(0.96)$ \\
5. Have you felt constantly overwhelmed and stressed? & $2.29(0.80)$ \\
6. Have you had the feeling that you cannot overcome your difficulties? & $3.08(0.91)$ \\
7. Have you been able to enjoy your normal daily activities? & $2.52(1.01)$ \\
8. Have you been able to adequately cope with problems? & $2.92(0.89)$ \\
9. Have you felt unhappy or depressed? & $2.47(0.79)$ \\
10. Have you lost confidence in yourself? & $2.73(1.01)$ \\
11. Have you thought that you are a worthless person? & $2.15(1.08)$ \\
GHQ-12 (over 12 points) & $1.68(1.01)$ \\
Cut-off point $\geq 3$ & $2.37(0.85)$ \\
Yes $30.16(3.76)$ \\
\hline No feel reasonably happy considering all the circumstances?
\end{tabular}

$\alpha$-Cronbach $=0.910$ Items 1 to 12 rank $1-4$.

As shown in Table 2, the items with the highest score are: Have you felt constantly overwhelmed and stressed? $\mathrm{M}=3.08(\mathrm{SD}=0.91)$; Have you been able to enjoy your normal daily 
activities? $\mathrm{M}=2.92(\mathrm{SD}=0.89)$; Have you been able to concentrate well on what you were doing? $\mathrm{M}=2.86$ ( $\mathrm{SD}=0.80)$; and Have your worries made you lose a lot of sleep? $\mathrm{M}=2.82(\mathrm{SD}=0.99)$.

On the contrary, the items with a lower valuation have been: Have you thought that you are a worthless person? $\mathrm{M}=1.68$ ( $\mathrm{SD}=1.01$ ); Do you feel reasonably happy considering all the circumstances? $\mathrm{M}=2.37(\mathrm{SD}=0.85)$ and Have you felt capable of making decisions? $\mathrm{M}=2.29$ $(\mathrm{SD}=0.80)$.

\subsection{Sociodemographic Data and Their Relationship with Psychological Distress}

Table 1 shows how PD is more present among women $\mathrm{OR}=1.916,95 \% \mathrm{CI}=(1.613$, $2.273)$; aged 29 or younger $\mathrm{OR}=2.651,95 \% \mathrm{CI}=(2.219,3.168)$; without a couple $\mathrm{OR}=1.959$, $95 \% \mathrm{CI}=(1.643,2.336)$; with lower educational level (secondary school or lower) $\mathrm{OR}=2.132$, $95 \% \mathrm{CI}=(1.771,2.567) ;$ living in a house without balcony / terrace/yard/garden OR $=1.631$, $95 \% \mathrm{CI}=(1.232,2.160)$; without children $\mathrm{OR}=2.222,95 \% \mathrm{CI}=(1.873,2.639)$; and not being a health professional $\mathrm{OR}=1.385,95 \% \mathrm{CI}=(1.062,1.580)$. Higher $\mathrm{PD}$ was found among public employees $(78.7 \%)$ than among workers of private companies $(70.6 \%)$ and self-employed workers $(61.8 \%), p<0.001$.

It is not observed that having a pet or any degree of disability is associated with the development of PD. Being in strict confinement or being able to go out only for purchase or work is indeed associated with the level of PD (Table 1).

\subsection{Physical Symptoms, Perception of Health, Health-Related Variables and Psychological Distress}

$76.8 \%$ claimed to have an excellent self-perceived health. A bad perception, versus an excellent health perception, is associated with a higher level of $\mathrm{PD}$, with an $\mathrm{OR}=6.803$, $95 \% \mathrm{CI}=(4.808,9.524)$ (Table 3$)$.

Table 3. Association between self-perceived health, physical symptoms, and other health variables related with psychological distress during the COVID-19 pandemic.

\begin{tabular}{|c|c|c|c|c|c|c|}
\hline \multicolumn{7}{|c|}{ TOTAL $(N=3227)$} \\
\hline \multicolumn{7}{|c|}{ GHQ } \\
\hline & $N(\%)$ & $\begin{array}{c}\text { YES } \\
(N=2544)\end{array}$ & $\begin{array}{c}\text { NO } \\
(N=683)\end{array}$ & $\chi^{2}$ & $p$ & $\begin{array}{c}\text { Odds Ratio } \\
(\mathrm{CI}=95 \%)\end{array}$ \\
\hline \multicolumn{7}{|c|}{ PHYSICAL SYMPTOMS } \\
\hline $\begin{array}{c}\text { Fever } \\
\text { Yes } \\
\text { No }\end{array}$ & $\begin{array}{c}46(1.4) \\
3181(98.6)\end{array}$ & $\begin{array}{l}89.1 \\
78.7\end{array}$ & $\begin{array}{l}10.9 \\
21.3\end{array}$ & 2.965 & 0.085 & $\begin{array}{c}2.221 \\
(0.874,5.643)\end{array}$ \\
\hline $\begin{array}{l}\text { Cough } \\
\text { Yes } \\
\text { No }\end{array}$ & $\begin{array}{c}647(20.0) \\
2580(80.0)\end{array}$ & $\begin{array}{l}85.3 \\
77.2\end{array}$ & $\begin{array}{l}14.7 \\
22.8\end{array}$ & 20.378 & $<0.001$ & $\begin{array}{c}1.715 \\
(1.354,2.172)\end{array}$ \\
\hline $\begin{array}{c}\text { Headache } \\
\text { Yes } \\
\text { No }\end{array}$ & $\begin{array}{l}1731(53.6) \\
1496(46.4)\end{array}$ & $\begin{array}{l}87.5 \\
68.8\end{array}$ & $\begin{array}{l}12.5 \\
31.2\end{array}$ & 168.868 & $<0.001$ & $\begin{array}{c}3.183 \\
(2.660,3.810)\end{array}$ \\
\hline $\begin{array}{c}\text { Muscle pain } \\
\text { Yes } \\
\text { No }\end{array}$ & $\begin{array}{c}845(26.2) \\
2382(73.8)\end{array}$ & $\begin{array}{l}89.0 \\
75.2\end{array}$ & $\begin{array}{l}11.0 \\
24.8\end{array}$ & 70.810 & $<0.001$ & $\begin{array}{c}2.662 \\
(2.105,3.366)\end{array}$ \\
\hline $\begin{array}{c}\text { Dizziness } \\
\text { Yes } \\
\text { No }\end{array}$ & $\begin{array}{c}363(11.2) \\
2864(88.8)\end{array}$ & $\begin{array}{l}90.9 \\
77.3\end{array}$ & $\begin{array}{c}9.1 \\
22.7\end{array}$ & 35.737 & $<0.001$ & $\begin{array}{c}2.936 \\
(2.031,4.243)\end{array}$ \\
\hline $\begin{array}{c}\text { Diarrhea } \\
\text { Yes } \\
\text { No }\end{array}$ & $\begin{array}{c}379(11.7) \\
2848(88.3)\end{array}$ & $\begin{array}{l}91.6 \\
77.1\end{array}$ & $\begin{array}{c}8.4 \\
22.9\end{array}$ & 41.655 & $<0.001$ & $\begin{array}{c}3.213 \\
(2.214,4.663)\end{array}$ \\
\hline $\begin{array}{c}\text { Sore throat } \\
\text { Yes } \\
\text { No }\end{array}$ & $\begin{array}{c}625(19.4) \\
2602(80.6)\end{array}$ & $\begin{array}{l}86.2 \\
77.1\end{array}$ & $\begin{array}{l}13.8 \\
22.9\end{array}$ & 25.474 & $<0.001$ & $\begin{array}{c}1.866 \\
(1.460,2.385)\end{array}$ \\
\hline
\end{tabular}


Table 3. Cont.

\begin{tabular}{|c|c|c|c|c|c|c|}
\hline \multicolumn{7}{|c|}{ TOTAL $(N=3227)$} \\
\hline \multicolumn{7}{|c|}{ GHQ } \\
\hline & $N(\%)$ & $\begin{array}{c}\text { YES } \\
(N=2544)\end{array}$ & $\begin{array}{c}\text { NO } \\
(N=683)\end{array}$ & $x^{2}$ & $p$ & $\begin{array}{c}\text { Odds Ratio } \\
(\mathrm{CI}=95 \%)\end{array}$ \\
\hline $\begin{array}{l}\text { Coryza } \\
\text { Yes } \\
\text { No }\end{array}$ & $\begin{array}{l}1122(34.8) \\
2105(65.2)\end{array}$ & $\begin{array}{l}85.6 \\
75.2\end{array}$ & $\begin{array}{l}14.4 \\
24.8\end{array}$ & 46.644 & $<0.001$ & $\begin{array}{c}1.949 \\
(1.606,2.366)\end{array}$ \\
\hline $\begin{array}{l}\text { Chills } \\
\text { Yes } \\
\text { No }\end{array}$ & $\begin{array}{c}225(7.0) \\
3002(93.0)\end{array}$ & $\begin{array}{l}88.4 \\
78.1\end{array}$ & $\begin{array}{l}11.6 \\
21.9\end{array}$ & 13.386 & $<0.001$ & $\begin{array}{c}2.144 \\
(1.412,3.256)\end{array}$ \\
\hline $\begin{array}{l}\text { Breathing difficulty } \\
\text { Yes } \\
\text { No }\end{array}$ & $\begin{array}{c}141(4.4) \\
3086(95.6)\end{array}$ & $\begin{array}{l}88.7 \\
78.4\end{array}$ & $\begin{array}{l}11.3 \\
21.6\end{array}$ & 8.517 & 0.004 & $\begin{array}{c}2.154 \\
(1.271,3.650)\end{array}$ \\
\hline \multicolumn{7}{|c|}{ CURRENT HEALTH STATUS } \\
\hline $\begin{array}{l}\text { Self-perceived health } \\
\text { Fair/bad/very bad } \\
\text { Excellent/good/very good }\end{array}$ & $\begin{array}{c}749(23.2) \\
2478(76.8)\end{array}$ & $\begin{array}{l}95.1 \\
73.9\end{array}$ & $\begin{array}{c}4.9 \\
26.1\end{array}$ & 153.894 & $<0.001$ & $\begin{array}{c}6.803 \\
(4.808,9.524)\end{array}$ \\
\hline $\begin{array}{c}\text { Chronic diseases } \\
\text { Yes } \\
\text { No }\end{array}$ & $\begin{array}{c}945(29.3) \\
2282(70.7)\end{array}$ & $\begin{array}{l}78.1 \\
79.1\end{array}$ & $\begin{array}{l}21.9 \\
20.9\end{array}$ & 0.438 & 0.508 & $\begin{array}{c}1.067 \\
(0.888,1.283)\end{array}$ \\
\hline $\begin{array}{c}\text { Currently taking any medication } \\
\text { No } \\
\text { Yes }\end{array}$ & $\begin{array}{l}2026(62.8) \\
1201(37.2)\end{array}$ & $\begin{array}{l}79.3 \\
78.0\end{array}$ & $\begin{array}{l}20.7 \\
22.0\end{array}$ & 0.764 & 0.382 & $\begin{array}{c}1.083 \\
(0.911,1.289)\end{array}$ \\
\hline $\begin{array}{c}\text { Hospitalised in the last } 14 \text { days } \\
\text { No } \\
\text { Yes }\end{array}$ & $\begin{array}{c}3208(99.4) \\
19(0.6)\end{array}$ & $\begin{array}{l}78.9 \\
73.7\end{array}$ & $\begin{array}{l}21.1 \\
26.3\end{array}$ & 0.304 & 0.581 & $\begin{array}{c}1.333 \\
(0.479,3.717)\end{array}$ \\
\hline $\begin{array}{c}\text { Health care in the last } 14 \text { days } \\
\text { Yes } \\
\text { No }\end{array}$ & $\begin{array}{c}281(8.7) \\
2946(91.3)\end{array}$ & $\begin{array}{l}82.6 \\
78.5\end{array}$ & $\begin{array}{l}17.4 \\
21.5\end{array}$ & 2.563 & 0.109 & $\begin{array}{c}1.291 \\
(0.937,1.779)\end{array}$ \\
\hline
\end{tabular}

The most frequent symptoms were: headache $(53.6 \%)$, rhinitis $(34.8 \%)$, muscle pain $(26.2 \%)$, cough $(20.0 \%)$, and sore throat $(19.4 \%)$. Having any of the symptoms is associated with developing PD, except for fever $(1.4 \%$ had it). The symptoms with the highest percentages of high PD are: diarrhea $(91.6 \%)$, dizziness $(90.9 \%)$, fever $(89.1 \%)$, muscle pain $(89.0 \%)$, breathing difficulties $(88.7 \%)$, chills $(88.4 \%)$, and headache $(87.5 \%)$; all results had $p<0.001$ (Table 3).

$29.3 \%$ reported having a chronic disease and $37.2 \%$ were taking medication. During the last 14 days, $8.7 \%$ had required medical care and $0.6 \%$ had required hospital care. No association was observed between these variables and developing PD.

\subsection{Psychological Distress and Contact History}

The proportion of participants who claimed they had not been in contact for more than $15 \mathrm{~min}$ and within $2 \mathrm{~m}$ away with an infected person was $78.1 \% .71 .9 \%$ reported not having been in casual contact with an infected person and $61.3 \%$ had not had any contact with a person or material suspected of being infected. $14.3 \%$ stated that they had undergone a diagnostic test (Table 4).

These contact histories, except for having received a diagnostic test, were associated with having developed PD, finding statistical significance $(p<0.05)$ and an OR greater than 1.3 (Table 4). 
Table 4. Association between variables related with history of contact and psychological distress during the pandemic.

\begin{tabular}{|c|c|c|c|c|c|c|}
\hline & & & $\begin{array}{l}\text { TOTAL } \\
N=3227)\end{array}$ & & \multirow[b]{3}{*}{$p$} & \multirow[b]{3}{*}{$\begin{array}{c}\text { Odds Ratio } \\
(\mathrm{CI}=95 \%)\end{array}$} \\
\hline & \multicolumn{4}{|c|}{ GHQ } & & \\
\hline & $N(\%)$ & $\begin{array}{c}\text { Yes } \\
(N=2544)\end{array}$ & $\begin{array}{c}\text { No } \\
(N=683)\end{array}$ & Statistical & & \\
\hline $\begin{array}{c}\text { Contact }>15^{\prime}<2 \text { m with infected person } \\
\text { Yes, or doesn't know } \\
\text { No }\end{array}$ & $\begin{array}{c}708(21.9) \\
2519(78.1)\end{array}$ & $\begin{array}{l}82.8 \\
77.7\end{array}$ & $\begin{array}{l}17.2 \\
22.3\end{array}$ & 8.411 & 0.004 & $\begin{array}{c}1.372 \\
(1.105,1.704)\end{array}$ \\
\hline $\begin{array}{c}\text { Casual contact with infected person } \\
\text { Yes, or doesn't know } \\
\text { No }\end{array}$ & $\begin{array}{c}907(28.1) \\
2320(71.9)\end{array}$ & $\begin{array}{l}83.0 \\
77.2\end{array}$ & $\begin{array}{l}17.0 \\
22.8\end{array}$ & 13.250 & $<0.001$ & $\begin{array}{c}1.453 \\
(1.190,1.772)\end{array}$ \\
\hline $\begin{array}{c}\text { Contact with any person or material } \\
\text { suspicious of being infected } \\
\text { Yes, or doesn't know } \\
\text { No }\end{array}$ & $\begin{array}{l}1248(38.7) \\
1979(61.3)\end{array}$ & $\begin{array}{l}82.8 \\
76.4\end{array}$ & $\begin{array}{l}17.2 \\
23.6\end{array}$ & 18.910 & $<0.001$ & $\begin{array}{c}1.485 \\
(1.240,1.777)\end{array}$ \\
\hline $\begin{array}{l}\text { Any infected family member } \\
\text { Yes, or doesn't know } \\
\text { No }\end{array}$ & $\begin{array}{c}537(16.6) \\
2690(83.4)\end{array}$ & $\begin{array}{l}82.5 \\
78.1\end{array}$ & $\begin{array}{l}17.5 \\
21.9\end{array}$ & 5.173 & 0.023 & $\begin{array}{c}1.320 \\
(1.038,1.679)\end{array}$ \\
\hline $\begin{array}{c}\text { Having been performed diagnostic test } \\
\text { No } \\
\text { Yes }\end{array}$ & $\begin{array}{c}2767(85.7) \\
460(14.3)\end{array}$ & $\begin{array}{l}79.2 \\
76.7\end{array}$ & $\begin{array}{l}20.8 \\
23.3\end{array}$ & 1.412 & 0.235 & $\begin{array}{c}1.153 \\
(0.912,1.460)\end{array}$ \\
\hline
\end{tabular}

\subsection{Psychological Distress and Preventive Measures}

The preventive measure with a higher mean score was "wearing a mask regardless of the presence of symptoms" $(\mathrm{M}=4.77$; $\mathrm{SD}=0.62)$, followed by "washing hands with soap and water" $(\mathrm{M}=4.75 ; \mathrm{SD}=0.52)$. A statistically significant association has been found between having PD and the use of the following preventive measures: "washing hands after coughing, touching the nose, or sneezing"; "avoiding sharing utensils", both with $p<0.001$; "leaving at least one and a half metres away", $p=0.002$; "wash hands with soap and water", $p=0.003$; and "washing hands with soap and water", $p=0.025$ (Table 5).

Table 5. Contrast between preventive measures and psychological distress during the pandemic.

\begin{tabular}{|c|c|c|c|c|c|}
\hline & \multicolumn{5}{|c|}{ TOTAL $(N=3227)$} \\
\hline & \multicolumn{4}{|c|}{ GHQ } & \multirow[b]{2}{*}{$p$} \\
\hline & $\mathrm{M}(\mathrm{SD})$ & $\begin{array}{c}\text { Yes } \\
(N=2544)\end{array}$ & $\begin{array}{c}\text { No } \\
(N=683)\end{array}$ & Statistical & \\
\hline Covering mouth & $4.56(0.76)$ & $4.55(0.76)$ & $4.60(0.75)$ & -1.411 & 0.158 \\
\hline Avoiding sharing utensils & $4.29(1.16)$ & $4.25(1.17)$ & $4.43(1.10)$ & -3.784 & $<0.001$ \\
\hline Washing hands with soap and water & $4.75(0.52)$ & $4.74(0.53)$ & $4.79(0.47)$ & -2.250 & 0.025 \\
\hline Washing hands with hydroalcoholic solution & $3.89(1.12)$ & $3.86(1.12)$ & $4.00(1.12)$ & -2.994 & 0.003 \\
\hline $\begin{array}{l}\text { Washing hands immediately after coughing, } \\
\text { touching the nose, or sneezing }\end{array}$ & $3.60(1.18)$ & $3.56(1.17)$ & $3.75(1.18)$ & -3.675 & $<0.001$ \\
\hline $\begin{array}{c}\text { Washing hands after touching potentially } \\
\text { contaminated objects }\end{array}$ & $4.57(0.75)$ & $4.56(0.76)$ & $4.62(0.72)$ & -1.710 & 0.087 \\
\hline $\begin{array}{c}\text { Wearing a mask regardless of the presence of } \\
\text { symptoms }\end{array}$ & $4.77(0.62)$ & $4.77(0.62)$ & $4.77(0.61)$ & -0.303 & 0.762 \\
\hline Leaving at least a metre and a half distance & $4.51(0.67)$ & $4.49(0.68)$ & $4.58(0.65)$ & -3.157 & 0.002 \\
\hline
\end{tabular}

Note: Likert-type response scale from 1 (Never) to 5 (Always).

\subsection{Prediction of Psychological Distress during the Pandemic}

The variables that can predict PD during the COVID-19 pandemic in Chile according to the binary logistic regression are: having a bad self-perception of health $\mathrm{OR}=4.038$, 
$95 \% \mathrm{CI}=(2.831,5.758)$; being younger than $29 \mathrm{OR}=2.287,95 \% \mathrm{CI}=(1.893,2.762)$; having diarrhea $\mathrm{OR}=2.093,95 \% \mathrm{CI}=(1.414,3.098)$; having headache $\mathrm{OR}=2.019,95 \% \mathrm{CI}=(1.662$, 2.453); being a woman $\mathrm{OR}=1.638,95 \% \mathrm{CI}=(1.363,1.967)$; having muscle pain $\mathrm{OR}=1.439$, $95 \% \mathrm{CI}=(1.114,1.859)$, and having had casual contact with an infected person OR $=1.410$, $95 \% \mathrm{CI}=(1.138,1.747)$.

These variables correctly predict and classify $79.5 \%$ of psychological distress, with a sensitivity/specificity of $17.4 / 96.2, \mathrm{R} 2=0.126$; Hosmer-Lemoshov test $\chi 2=13.514$ $(p=0.095)$ and Omnibus test $\chi 2=433.575(p<0.001)$.

\section{Discussion}

As previously mentioned, the development of psychological effects, especially PD, is an event that is closely related to the occurrence of the COVID-19 pandemic. In the present study, a high percentage of people with a high level of PD (78.83\%) has been observed, with a $\geq 3$ cut-off point in the GHQ-12, data that are above those obtained in Spain (71.98\%) with a similar study methodology and cut-off point [20]. The choice of cut-off point at this given level $(\geq 3)$ should be considered when comparing with other studies. These results are consistent with those obtained in previous similar studies [43-45].

It has been suggested that once key responses are adopted at the public health level, such as diagnostic testing, contact tracking, lockdown, and the management of confirmed cases of COVID-19, perhaps it is time to prioritize measures to safeguard the mental health of Chileans [46]. Even more, with the knowledge that the percentage of the population fully vaccinated has achieved higher levels than in other countries of the same geographical environment [13]. However, the speed in vaccinating the population may have caused an unjustified optimism that led to the abandonment of preventive measures after the first dose of the vaccine and, as the PAHO Director stated, "the vaccine alone is not going to stop the pandemic" [47].

Therefore, it seems that there is still time to prevent serious effects on mental health, since studies conducted in 21 countries, including Chile, have not observed, for instance, that high levels of PD, as the ones found in the study at hand, have led to an increase in the number of suicides in the first months of the pandemic [35].

In a somewhat contradictory way, a high percentage of the studied population claims to have an excellent self-perception of health during the last 14 days $(76.8 \%)$, being the variable that mostly predicts PD, in the same way that it is known that PD is a highly reliable predictor of mortality [42]. This leads to a certain degree of optimism if measures are taken in time to enhance protective factors and mitigate the effects of the foreseeable economic recession resulting from this health crisis [35].

Differences have been seen between the symptoms found in a group of Latin American countries, the most common being cough $(60.1 \%)$, fatigue/tiredness $(52.0 \%)$, sore throat $(50.3 \%)$, and fever $(44.2 \%)$ [16], while in the present study, the most frequent symptoms have been: headache $(53.6 \%)$, rhinitis $(34.8 \%)$, muscle pain $(26.2 \%)$, cough $(20.0 \%)$, and sore throat (19.4\%). In a study carried out in Spain with the same methodology, both headache and muscle pain had similar figures, but sore throat and cough had significantly higher values, while rhinitis occurred at higher rates in Chile [40]. This difference could be explained to some extent by the sociodemographic variables, the different information received by the populations of these countries, or even by being in different climatic seasons derived from belonging to the northern or southern hemisphere. In the study at hand, the three symptoms that predict PD are diarrhea, headache, and muscle pain.

The sex and age variables, as well as living with children, predict the level of PD, as has been also referred to in the literature [24,28,29,38,48-51].

Having a history of contact is associated with the presence of PD, both through contact with contaminated people or material, or with infected relatives, but it is the variable "having been in casual contact with an infected person" that mostly predicts PD, something already corroborated in previous studies [27]. 
It is well known that the proper and early use of preventive measures to avoid COVID19 produces benefits in terms of health [52]. In this sense, the preventive measures with a higher valuation are: "wearing a mask regardless of the presence of symptoms" and "washing hands with soap and water". This second measure coincides with the study carried out in Spain [20], but the use of a mask receives a much higher value in Chile. This could be explained by the fact that the data collection was carried out in Spain in earlier dates than in Chile, and during the first months of the pandemic, in Spain there was no such recommendation for the widespread use of the mask, and there were even supply problems.

On the other hand, in other studies carried out in Chile, it was found that males and people under 60 years of age were the most compliant groups with the preventive measures established by the Government, while in Colombia or Ecuador, it was women and the elderly who complied the most [48].

When designing public health policies, the stressors identified in the literature, which are related to financial, academic, and family concerns, should be taken into account, the stress of confinement being a clear predictor of mental health [24], and obviously influencing the conditions of the home of the confined person. In the present study, we have found that PD is associated with living in houses with no exterior exit (house without a balcony, terrace, yard, or garden), identifying this as the most potentially stressful type of housing, an issue that would be convenient to consider when planning urban development.

Living without a partner and not having children are other stressor variables identified in this study, which highlight the importance of family support in pandemic situations, as the importance of social support has been observed with other health problems [53].

Compared with all six Latin American countries that have been studied (Argentina, Brazil, Chile, Colombia, Mexico, and Peru), Chile is the country shown to have positive variables against COVID-19, since it has a lower percentage of poverty, higher level of schooling, and the best health system. On the contrary, it has a high percentage of the population over 65 years of age, and it adopted a partial and not total lockdown, unlike other surrounding countries [54]. Another positive factor is the fast pace of vaccination administration, higher not only than other Latin American countries, but even higher than some European countries, such as Spain [13].

On the other hand, health professionals, as previously mentioned, are a group with high levels of PD and other indicators of poor mental health $[16,33]$, while in the present study, show a lower percentage of PD than non-health professionals. One possible explanation may be the invisibility of non-health workers in situations of risk (e.g., delivery staff, cleaners, drivers, law enforcement bodies), who, being essential jobs, have had to continue performing their work during lockdown, being in contact with contaminated people or objects and for whom vaccination has not been established as a priority, as has been the case with health professionals. Other explanations may be the effect of teleworking, increased lockdown, or greater effects on the economy, with its consequent impact on PD [55] or the social support they have had during the pandemic [56], although the latter has not been observed in other studies [57].

In this socio-economic context, and based on the levels of PD found in the study, the adoption of preventive measures focused on the prevention of possible mental effects in high-risk populations is considered of special relevance.

The limitations of this research are the same as those of all descriptive studies without randomized sampling, along with those related to online access to data, which leave out groups without internet access or without knowledge for its use. This can be seen in that $59.25 \%$ of the participants had a university level of education or higher. Moreover, online data collection does not guarantee a homogeneous territorial distribution, with some areas of the country being more affected than others. However, the characteristics of the study advised applying the sampling used. In addition, this research has been carried out with the same methodology in 18 countries in Latin America and Europe, which will facilitate comparisons in the near future that will allow increasing the available evidence on the issue 
studied. This methodology was also chosen for the Eurofound study and promoted by the European Union [40], but its results did not allow for causal associations to be obtained and will require future studies with more appropriate designs to test the hypotheses detected. Another limitation is the difficulty in answering certain questions, such as "whether having touched contaminated objects" or "having been in contact with sick people during the previous 14 days". Similarly, the GHQ is a general measure of mental health, although it is a widely used and highly reliable indicator. In our study, it obtained an $\alpha$-Cronbach's score $=0.910$.

\section{Conclusions}

We have been able to verify that in Chile, a country with theoretically high levels of protection against the COVID-19 pandemic, including a high percentage of vaccination, higher than those of neighboring countries, the percentage of people with psychological distress is very high in the population studied.

It has been possible to identify variables associated with PD such as being a woman, being under 29 years of age, and with a low level of education, vulnerable groups already described in other countries. Furthermore, the influence of family support becomes visible by observing that living without a partner or not having children act as variables associated with PD. The type of housing is another factor to consider when it comes to urban planning, and to establish the importance of having a house with exterior exit (balcony/terrace/yard) to reduce the PD generated in pandemics that force diverse degrees of lockdown of the population.

Something seemingly contradictory has been detected; non-health professionals showed a higher level of PD than health professionals, a group that is the subject of most studies regarding the effects of the COVID-19 pandemic. This can help visualize the group of workers of essential activities, who have had to continue to develop their work during the pandemic and, therefore, have also been exposed to contact with contaminated people or objects, but with a lower level of prioritization when it comes to vaccination.

For these reasons, the need to prioritize the establishment of programs that safeguard the mental health of Chileans before these negative effects evolve into irremediable situations or become difficult to address is evident. In this study, vulnerable groups with whom intervention would be efficient and effective, have been identified.

Author Contributions: Conceptualization, C.R.-F., D.D.-G., M.O.-M., D.D.-B., D.E.-G., J.J.G.-I. and J.G.-S.; Data curation, C.R.-F., D.D.-G., M.O.-M., D.D.-B., D.E.-G., J.J.G.-I. and J.G.-S.; Formal analysis, C.R.-F., D.D.-G., M.O.-M., D.D.-B., D.E.-G., J.J.G.-I. and J.G.-S.; Investigation, C.R.-F., D.D.-G., M.O.M., D.D.-B., D.E.-G., J.J.G.-I. and J.G.-S.; Methodology, C.R.-F., D.D.-G., M.O.-M., D.D.-B., D.E.-G., J.J.G.-I. and J.G.-S.; Project administration, C.R.-F. and J.G.-S.; Resources, C.R.-F., D.D.-G., M.O.-M., D.D.-B., D.E.-G., J.J.G.-I. and J.G.-S.; Software, C.R.-F., D.D.-G., M.O.-M., D.D.-B., D.E.-G., J.J.G.-I. and J.G.-S.; Supervision, C.R.-F., D.D.-G., M.O.-M., D.D.-B., D.E.-G., J.J.G.-I. and J.G.-S.; Validation, C.R.-F., D.D.-G., M.O.-M., D.D.-B., D.E.-G., J.J.G.-I. and J.G.-S.; Visualization, C.R.-F., D.D.-G., M.O.-M., D.D.B., D.E.-G., J.J.G.-I. and J.G.-S.; Writing-original draft, C.R.-F., D.D.-G., M.O.-M., D.D.-B., D.E.-G., J.J.G.-I. and J.G.-S.; Writing-review \& editing, C.R.-F., D.D.-G., M.O.-M., D.D.-B., D.E.-G., J.J.G.-I. and J.G.-S. All authors have read and agreed to the published version of the manuscript.

Funding: This research received no external funding.

Institutional Review Board Statement: The study was conducted according to the guidelines of the Declaration of Helsinki and has been authorized by the Ethics Committee of the University of Aconcagua in Chile (Santiago, UAC-22 April 2020) and in Spain by the Research Ethics Committee of Huelva, belonging to the Regional Ministry of Health of Andalusia (PI 036/20).

Informed Consent Statement: Informed consent was obtained from all subjects involved in the study.

Data Availability Statement: All data are available within this article.

Acknowledgments: In memory of Guillermo Solar Oyanedel. 
Conflicts of Interest: The authors declare no conflict of interest.

\section{References}

1. WHO. Europe International Health Regulations-2019-nCoV Outbreak Is an Emergency of International Concern. Available online: https:/ / www.who.int/director-general/speeches/detail/who-director-general-s-statement-on-ihr-emergencycommittee-on-novel-coronavirus-(2019-ncov) (accessed on 21 May 2021).

2. World Health Organization. WHO Announces COVID-19 Outbreak a Pandemic. 2020. Available online: https://www.euro.who. $\mathrm{int/en/health-topics/health-emergencies} \mathrm{/} \mathrm{coronavirus-covid-19/news/news} \mathrm{/2020/3/who-announces-covid-19-outbreak-a-}$ pandemic (accessed on 21 May 2021).

3. Newey, S. Latin America Has Become the Epicentre of the Coronavirus Pandemic, WHO Says, The Telegraph, 27 May 2020. Available online: https:/ / www.telegraph.co.uk/global-health/science-and-disease/latin-america-has-become-epicentre-coronaviruspandemic-says/ (accessed on 21 May 2021).

4. Benítez, M.A.; Velasco, C.; Sequeira, A.R.; Henríquez, J.; Menezes, F.M.; Paolucci, F. Responses to COVID-19 in five Latin American countries. Health Policy Technol. 2020, 9, 525-559. [CrossRef] [PubMed]

5. Bilal, U.; Alfaro, T.; Vives, A. COVID-19 and the worsening of health inequities in Santiago, Chile. Int. J. Epidemiol. 2021, dyab007. [CrossRef]

6. Villalobos-Dintrans, P.; Castillo, C.; de la Fuente, F.; Maddaleno, M. COVID-19 incidence and mortality in the Metropolitan Region, Chile: Time, space, and structural factors. PLoS ONE 2021, 16, e0250707. [CrossRef] [PubMed]

7. Mena, G.; Martinez, P.P.; Mahmud, A.S.; Marquet, P.A.; Buckee, C.O.; Santillana, M. Socioeconomic status determines COVID-19 incidence and related mortality in Santiago, Chile. MedRxiv 2021. [CrossRef]

8. Asahi, K.; Undurraga, E.A.; Valdés, R.; Wagner, R. The effect of COVID-19 on the economy: Evidence from an early adopter of localized lockdowns. J. Glob. Health 2021, 11, 05002. [CrossRef] [PubMed]

9. Ramírez de la Cruz, E.E.; Grin, E.J.; Sanabria-Pulido, P.; Cravacuore, D.; Orellana, A. The Transaction Costs of the Governments' Response to the COVID-19 Emergency in Latin America. Public Adm. Rev. 2020. [CrossRef] [PubMed]

10. Undurraga, E.A.; Chowell, G.; Mizumoto, K. COVID-19 case fatality risk by age and gender in a high testing setting in Latin America: Chile, March-August 2020. Infect. Dis. Poverty 2021, 10, 11. [CrossRef] [PubMed]

11. Millalen, P.; Nahuelpan, H.; Hofflinger, A.; Martínez, E. COVID-19 and Indigenous peoples in Chile: Vulnerability to contagion and mortality. AlterNative 2020, 16, 399-402. [CrossRef]

12. Del Pino, S.; Gómez-Salgado, J.; Domínguez-Gómez, J.A.; Ruiz-Frutos, C. People of african descent of the americas, racial discrimination, and quality of the health services. Sustainability 2020, 12, 6930. [CrossRef]

13. John Hopkins Coronavirus Resource Center. Available online: https://coronavirus.jhu.edu/region/chile (accessed on 18 May 2021).

14. Makaronidis, J.; Mok, J.; Balogun, N.; Magee, C.G.; Omar, R.Z.; Carnemolla, A.; Batterham, R.L. Seroprevalence of SARS-CoV-2 antibodies in people with an acute loss in their sense of smell and/or taste in a community-based population in London, UK: An observational cohort study. PLoS Med. 2020, 17, e1003358. [CrossRef] [PubMed]

15. Huang, C.; Huang, L.; Wang, Y.; Li, X.; Ren, L.; Gu, X.; Kang, L.; Guo, L.; Liu, M.; Zhou, X.; et al. 6-month consequences of COVID-19 in patients discharged from hospital: A cohort study. Lancet 2021, 397, 220-232. [CrossRef]

16. Luo, M.; Guo, L.; Yu, M.; Jiang, W.; Wang, H. The psychological and mental impact of coronavirus disease 2019 (COVID-19) on medical staff and general public-A systematic review and meta-analysis. Psychiatry Res. 2020, 291, 113190. [CrossRef] [PubMed]

17. Xiong, Q.; Xu, M.; Li, J.; Liu, Y.; Zhang, J.; Xu, Y.; Dong, W. Clinical sequelae of COVID-19 survivors in Wuhan, China: A single-centre longitudinal study. Clin. Microbiol. Infect. 2021, 27, 89-95. [CrossRef] [PubMed]

18. Kronbichler, A.; Kresse, D.; Yoon, S.; Lee, K.H.; Effenberger, M.; Shin, J.I. Asymptomatic patients as a source of COVID-19 infections: A systematic review and meta-analysis. Int. J. Infect. Dis. 2020, 98, 180-186. [CrossRef]

19. Ramírez, M.L.; Martinez, S.M.; Bessone, C.D.V.; Allemandi, D.A.; Quinteros, D.A. COVID-19: Epidemiological situation of Argentina and its neighbor countries after three months of pandemic. Disaster Med. Public Health Prep. 2021, 1-23. [CrossRef]

20. Domínguez-Salas, S.; Gómez-Salgado, J.; Andrés-Villas, M.; Díaz-Milanés, D.; Romero-Martín, M.; Ruiz-Frutos, C. PsychoEmotional Approach to the Psychological Distress Related to the COVID-19 Pandemic in Spain: A Cross-Sectional Observational Study. Healthcare 2020, 8, 190. [CrossRef]

21. Orte, C.; Sánchez-Prieto, L.; Domínguez, D.C.; Barrientos-Báez, A. Evaluation of Distress and Risk Perception Associated with COVID-19 in Vulnerable Groups. Int. J. Environ. Res. Public Health 2020, 17, 9207. [CrossRef]

22. Pollak, Y.; Shoham, R.; Dayan, H.; Gabrieli-Seri, O.; Berger, I. Background and concurrent factors predicting non-adherence to public health preventive measures during the chronic phase of the COVID-19 pandemic. J. Public Health 2021, fdab214. [CrossRef]

23. Galehdar, N.; Kamran, A.; Toulabi, T.; Heydari, H. Exploring nurses' experiences of psychological distress during care of patients with COVID-19: A qualitative study. BMC Psychiatry 2020, 20, 489. [CrossRef]

24. Landa-Blanco, M.; Mejía, C.J.; Landa-Blanco, A.L.; Martínez-Martínez, C.A.; Vásquez, D.; Vásquez, G.; Moraga-Vargas, P.; Echenique, Y.; Del Cid, G.M.; Montoya, B.D. Coronavirus awareness, confinement stress, and mental health: Evidence from Honduras, Chile, Costa Rica, Mexico and Spain. Soc. Sci. Med. 2021, 277, 113933. [CrossRef] 
25. Urzúa, A.; Samaniego, A.; Caqueo-Urízar, A.; Zapata Pizarro, A.; Irarrázaval Domínguez, M. Salud mental en trabajadores de la salud durante la pandemia por COVID-19 en Chile [Mental health problems among health care workers during the COVID-19 pandemic]. Rev. Med. Chil. 2020, 148, 1121-1127. (In Spanish) [CrossRef] [PubMed]

26. Giorgi, G.; Lecca, L.I.; Alessio, F.; Finstad, G.L.; Bondanini, G.; Lulli, L.G.; Arcangeli, G.; Mucci, N. COVID-19-Related Mental Health Effects in the Workplace: A Narrative Review. Int. J. Environ. Res. Public Health 2020, 17, 7857. [CrossRef] [PubMed]

27. Xiang, Y.T.; Yang, Y.; Li, W.; Zhang, L.; Zhang, Q.; Cheung, T.; Ng, C.H. Timely mental health care for the 2019 novel coronavirus outbreak is urgently needed. Lancet Psychiatry 2020, 7, 228-229. [CrossRef]

28. Dagnino, P.; Anguita, V.; Escobar, K.; Cifuentes, S. Psychological Effects of Social Isolation Due to Quarantine in Chile: An Exploratory Study. Front. Psychiatry 2020, 11, 591142. [CrossRef]

29. Ruiz-Frutos, C.; Ortega-Moreno, M.; Allande-Cussó, R.; Domínguez-Salas, S.; Dias, A.; Gómez-Salgado, J. Health-related factors of psychological distress during the COVID-19 pandemic among non-health workers in Spain. Saf. Sci. 2021, 133, 104996. [CrossRef]

30. Mohammadian Khonsari, N.; Shafiee, G.; Zandifar, A.; Mohammad Poornami, S.; Ejtahed, H.S.; Asayesh, H.; Qorbani, M. Comparison of psychological symptoms between infected and non-infected COVID-19 health care workers. BMC Psychiatry 2021, 21, 170. [CrossRef]

31. Pandey, D.; Bansal, S.; Goyal, S.; Garg, A.; Sethi, N.; Pothiyill, D.I.; Sreelakshmi, E.S.; Sayyad, M.G.; Sethi, R. Psychological impact of mass quarantine on population during pandemics-The COVID-19 Lock-Down (COLD) study. PLoS ONE 2020, 15, e0240501. [CrossRef]

32. Pappa, S.; Ntella, V.; Giannakas, T.; Giannakoulis, V.G.; Papoutsi, E.; Katsaounou, P. Prevalence of depression, anxiety, and insomnia among healthcare workers during the COVID-19 pandemic: A systematic review and meta-analysis. Brain Behav. Immun. 2020, 88, 901-907. [CrossRef]

33. Pfefferbaum, B.; North, C.S. Mental health and the COVID-19 pandemic. N. Engl. J. Med. 2020. [CrossRef]

34. Yao, H.; Chen, J.H.; Xu, Y.F. Patients with mental health disorders in the COVID-19 epidemic. Lancet Psychiatry 2020,7 , e21. [CrossRef]

35. Pirkis, J.; John, A.; Shin, S.; DelPozo-Banos, M.; Arya, V.; Analuisa-Aguilar, P.; Appleby, L.; Arensman, E.; Bantjes, J.; Baran, A.; et al. Suicide trends in the early months of the COVID-19 pandemic: An interrupted time-series analysis of preliminary data from 21 countries. Lancet Psychiatry 2021, 8, 579-588. [CrossRef]

36. Kim, K.S.; Kang, P.M.; Kim, H.S.N. Generalized Anxiety and Major Depressive Symptoms of General Public in South Korea during the Early COVID-19 Pandemic. Int. J. Ment. Health Promot. 2021, 23, 303-317. [CrossRef]

37. González-Tovar, M.; Hernández-Rodríguez, S. COVID-19 and Emotional Variables in a Sample of Chileans. Front. Psychol. 2021, 12, 615268. [CrossRef] [PubMed]

38. Gómez-Salgado, J.; Andrés-Villas, M.; Domínguez-Salas, S.; Díaz-Milanés, D.; Ruiz-Frutos, C. Related Health Factors of Psychological Distress During the COVID-19 Pandemic in Spain. Int. J. Environ. Res. Public Health 2020, 17, 3947. [CrossRef] [PubMed]

39. Tehrani, H. Mental health stigma related to novel coronavirus disease (COVID-19) in older adults. Geriatr. Gerontol. Int. 2020, 20, 796-797. [CrossRef] [PubMed]

40. Eurofound. Living, Working and COVID-19; COVID-19 Series; Publications Office of the European Union: Luxembourg, 2020.

41. Goldberg, D.P.; Gater, R.; Sartorius, N.; Ustun, T.B.; Piccinelli, M.; Gureje, O.; Rutter, C. The validity of two versions of the GHQ in the WHO study of mental illness in general health care. Psychol. Med. 1997, 27, 191-197. [CrossRef] [PubMed]

42. Idler, E.L.; Benyamini, Y. Self-rated health and mortality: A review of twenty-seven community studies. J. Health Soc. Behav. 1997, 38, 21-37. [CrossRef]

43. Dichter, M.N.; Sander, M.; Seismann-Petersen, S.; Köpke, S. COVID-19: It is time to balance infection management and personcentered care to maintain mental health of people living in German nursing homes. Int. Psychogeriatr. 2020, 32, 1157-1160. [CrossRef]

44. Si, M.Y.; Su, X.Y.; Jiang, Y.; Wang, W.J.; Gu, X.F.; Ma, L.; Li, J.; Zhang, S.K.; Ren, Z.F.; Ren, R.; et al. Psychological impact of COVID-19 on medical care workers in China. Infect. Dis. Poverty 2020, 9, 113. [CrossRef]

45. Serrano-Ripoll, M.J.; Meneses-Echavez, J.F.; Ricci-Cabello, I.; Fraile-Navarro, D.; Fiol-deRoque, M.A.; Pastor-Moreno, G.; Castro, A.; Ruiz-Pérez, I.; Zamanillo-Campos, R.; Gonçalves-Bradley, D.C. Impact of viral epidemic outbreaks on mental health of healthcare workers: A rapid systematic review and meta-analysis. J. Affect. Disord. 2020, 277, 347-357. [CrossRef]

46. Caqueo-Urízar, A.; Urzúa, A.; Aragón-Caqueo, D.; Charles, C.H.; El-Khatib, Z.; Otu, A.; Yaya, S. Mental health and the COVID-19 pandemic in Chile. Psychol. Trauma 2020, 12, 521-523. [CrossRef] [PubMed]

47. Taylor, L. COVID-19: Spike in cases in Chile is blamed on people mixing after first vaccine shot. BMJ 2021, 373, n1023. [CrossRef] [PubMed]

48. Bermejo-Martins, E.; Luis, E.O.; Sarrionandia, A.; Martínez, M.; Garcés, M.S.; Oliveros, E.Y.; Cortés-Rivera, C.; Belintxon, M.; Fernández-Berrocal, P. Different Responses to Stress, Health Practices, and Self-Care during COVID-19 Lockdown: A Stratified Analysis. Int. J. Environ. Res. Public Health 2021, 18, 2253. [CrossRef]

49. González-Sanguino, C.; Ausín, B.; Castellanos, M.Á.; Saiz, J.; López-Gómez, A.; Ugidos, C.; Muñoz, M. Mental health consequences during the initial stage of the 2020 Coronavirus pandemic (COVID-19) in Spain. Brain Behav. Immun. 2020, 87, 172-176. [CrossRef] [PubMed] 
50. Salazar-Fernández, C.; Palet, D.; Haeger, P.A.; Román Mella, F. COVID-19 Perceived Impact and Psychological Variables as Predictors of Unhealthy Food and Alcohol Consumption Trajectories: The Role of Gender and Living with Children as Moderators. Int. J. Environ. Res. Public Health 2021, 18, 4542. [CrossRef]

51. Skapinakis, P.; Bellos, S.; Oikonomou, A.; Dimitriadis, G.; Gkikas, P.; Perdikari, E.; Mavreas, V. Depression and Its Relationship with Coping Strategies and Illness Perceptions during the COVID-19 Lockdown in Greece: A Cross-Sectional Survey of the Population. Depress. Res. Treat. 2020, 2020, 3158954. [CrossRef]

52. Michael, G.; Vishan, N. Does Social Distancing Matter? (30 March 2020). University of Chicago, Becker Friedman Institute for Economics Working Paper No. 2020-26. SSRN Electron. J. 2020. [CrossRef]

53. Marroquín, B.; Vine, V.; Morgan, R. Mental health during the COVID-19 pandemic: Effects of stay-at-home policies, social distancing behavior, and social resources. Psychiatry Res. 2020, 293, 113419. [CrossRef]

54. Martinez-Valle, A. Public health matters: Why is Latin America struggling in addressing the pandemic? J. Public. Health Policy 2021, 42, 27-40. [CrossRef]

55. García-Iglesias, J.J.; Gómez-Salgado, J.; Martín-Pereira, J.; Fagundo-Rivera, J.; Ayuso-Murillo, D.; Martínez-Riera, J.R.; Ruiz-Frutos, C. Impacto del SARS-CoV-2 (COVID-19) en la salud mental de los profesionales sanitarios: Una revisión sistemática [Impact of SARS-CoV-2 (COVID-19) on the mental health of healthcare professionals: A systematic review.]. Rev. Esp. Salud. Publica. 2020, 94, e202007088. (In Spanish) [PubMed]

56. Peng, X.; Zhao, H.Z.; Yang, Y.; Rao, Z.L.; Hu, D.Y.; He, Q. Post-traumatic Growth Level and Its Influencing Factors Among Frontline Nurses During the COVID-19 Pandemic. Front. Psychiatry 2021, 12, 632360. [CrossRef] [PubMed]

57. Gómez-Salgado, J.; Ortega-Moreno, M.; Soriano, G.; Fagundo-Rivera, J.; Allande-Cussó, R.; Ruiz-Frutos, C. History of contact with the SARS-CoV-2 virus and the sense of coherence in the development of psychological distress in the occupational health professionals in Spain. Sci. Prog. 2021, 104, 368504211026121. [CrossRef] [PubMed] 